G. K. Sankaran and J. G. Spandaw

Nagoya Math. J.

Vol. 168 (2002), 113-125

\title{
THE MODULI SPACE OF BILEVEL-6 ABELIAN SURFACES
}

\author{
G. K. SANKARAN AND J. G. SPANDAW
}

\begin{abstract}
We show that the moduli space of abelian surfaces with polarisation of type $(1,6)$ and a bilevel structure has positive Kodaira dimension and indeed $p_{g} \geq 3$. To do this we show that three of the Siegel cusp forms with character for the paramodular symplectic group constructed by Gritsenko and Nikulin are cusp forms without character for the modular group associated to this moduli problem. We then calculate the divisors of the corresponding differential forms, using information about the fixed loci of elements of the paramodular group previously obtained by Brasch.
\end{abstract}

The moduli space $\mathcal{A}_{t}^{\text {bil }}$ of $(1, t)$-polarised abelian surfaces with a weak bilevel structure was introduced by S. Mukai in $[\mathrm{Mu}]$. Mukai showed that $\mathcal{A}_{t}^{\text {bil }}$ is rational for $t=2,3,4,5$. More generally, we may ask for birational invariants, such as Kodaira dimension, of a smooth model of a compactification of $\mathcal{A}_{t}^{\text {bil }}$ : since the choice of model does not affect birational invariants, we refer to the Kodaira dimension, etc., of $\mathcal{A}_{t}^{\text {bil }}$.

From the description of $\mathcal{A}_{t}^{\text {bil }}$ as a Siegel modular 3-fold $\Gamma_{t}^{\text {bil }} \backslash \mathbb{H}_{2}$ and the fact that $\Gamma_{t}^{\text {bil }} \subset \mathrm{Sp}(4, \mathbb{Z})$ it follows, by a result of L. Borisov [Bo], that $\kappa\left(\mathcal{A}_{t}^{\text {bil }}\right)=3$ for all sufficiently large $t$. For an effective result in this direction see [Sa]. In this note we shall prove an intermediate result for the case $t=6$.

Theorem A. The moduli space $\mathcal{A}_{6}^{\text {bil }}$ has geometric genus $p_{g}\left(\mathcal{A}_{6}^{\text {bil }}\right) \geq 3$ and Kodaira dimension $\kappa\left(\mathcal{A}_{6}^{\mathrm{bil}}\right) \geq 1$.

The case $t=6$ attracts attention for two reasons: it is the first case not covered by the results of [Mu]; and the image of the Humbert surface $\mathcal{H}_{1}(1)$ in $\mathcal{A}_{t}^{\text {bil }}$, which in the cases $2 \leq t \leq 5$ is a quadric and plays an important role both in $[\mathrm{Mu}]$ and below, becomes an abelian surface (at least birationally) because the modular curve $X(6)$ has genus 1 .

Received June 11, 2001.

2000 Mathematics Subject Classification: 11F46, 14K10. 
The method we use is that of Gritsenko, who proved a similar result for the moduli spaces of $(1, t)$-polarised abelian surfaces with canonical level structure for certain values of $t$ : see $[\mathrm{Gr}]$, especially Corollary 2. We use some of the weight 3 modular forms constructed by Gritsenko and Nikulin as lifts of Jacobi forms in [GN] to produce canonical forms having effective, nonzero, divisors on a suitable projective model $X_{6}$ of $\mathcal{A}_{6}^{\text {bil }}$. A similar method was used by Gritsenko and Hulek in [GH2] to give a new proof that the Barth-Nieto threefold is Calabi-Yau.

We also derive some information about divisors in $X_{6}$ and linear relations among them.

Acknowledgement. We are grateful to the DAAD and the British Council for financial assistance under ARC Project 313-ARC-XIII-99/45.

\section{§1. Compactification}

According to $[\mathrm{Mu}], \mathcal{A}_{t}^{\text {bil }}$ is isomorphic to the quotient $\Gamma_{t}^{\mathrm{bil}} \backslash \mathbb{H}_{2}$, where $\mathbb{H}_{2}$ is the Siegel upper half-plane $\left\{Z \in M_{2 \times 2}(\mathbb{C}) \mid Z={ }^{\top} Z, \operatorname{Im} Z>0\right\}$ and $\Gamma_{t}^{\text {bil }}=\Gamma_{t}^{\natural} \cup \zeta \Gamma_{t}^{\natural} \subset \operatorname{Sp}(4, \mathbb{Z})$ acts on $\mathbb{H}_{2}$ by fractional linear transformations. Here $\zeta=\operatorname{diag}(-1,1,-1,1)$ and, writing $\mathbf{I}_{n}$ for the $n \times n$ identity matrix,

$$
\Gamma_{t}^{\natural}=\left\{\gamma \in \operatorname{Sp}(4, \mathbb{Z}) \mid \gamma-\mathbf{I}_{4} \in\left(\begin{array}{cccc}
t \mathbb{Z} & \mathbb{Z} & t \mathbb{Z} & t \mathbb{Z} \\
t \mathbb{Z} & t \mathbb{Z} & t \mathbb{Z} & t^{2} \mathbb{Z} \\
t \mathbb{Z} & \mathbb{Z} & t \mathbb{Z} & t \mathbb{Z} \\
\mathbb{Z} & \mathbb{Z} & \mathbb{Z} & t \mathbb{Z}
\end{array}\right)\right\} .
$$

We define $H(\mathbb{Z})$ to be the Heisenberg group $\mathbb{Z} \rtimes \mathbb{Z}^{2}$ embedded in $\operatorname{Sp}(4, \mathbb{Z})$ as

$$
H(\mathbb{Z})=\left\{[m, n ; k]=\left(\begin{array}{cccc}
1 & m & 0 & 0 \\
0 & 1 & 0 & 0 \\
0 & n & 1 & 0 \\
n & k & -m & 1
\end{array}\right) \mid m, n, k \in \mathbb{Z}\right\} .
$$

LEMMA 1.1. $\Gamma_{6}^{\natural}$ is neat; that is, if $\lambda$ is an eigenvalue of some $\gamma \in \Gamma_{6}^{\natural}$ which is a root of unity, then $\lambda=1$. Any torsion element of $\Gamma_{6}^{\text {bil }}$ has order 2 and fixes a divisor in $\mathbb{H}_{2}$.

Proof. Suppose that $\gamma \in \Gamma_{6}^{\natural}$ : then the characteristic polynomial of $\gamma$ is congruent to $(1-x)^{4} \bmod 6$. If some $\gamma \in \Gamma_{6}^{\natural}$ has an eigenvalue $\lambda$ which 
is a nontrivial root of unity, then we may assume that $\lambda$ is a primitive $p$ th root of unity for some prime $p$. The minimum polynomial $m_{\lambda}(x)$ of $\lambda$ over $\mathbb{Z}$ divides the characteristic polynomial of $\gamma$; so $p=2,3$ or 5 , since $\operatorname{deg} m_{\lambda}=p-1$. But then $m_{\lambda}(x)=1+x, 1+x+x^{2}$ or $1+x+x^{2}+x^{3}+x^{4}$. The second of these does not divide $(1-x)^{4}$ in $\mathbb{F}_{2}[x]$ and the other two do not divide $(1-x)^{4}$ in $\mathbb{F}_{3}[x]$.

So any torsion element of $\Gamma_{6}^{b i l}$ is of the form $\gamma=\zeta \gamma^{\prime}$ for some $\gamma^{\prime} \in \Gamma_{6}^{\natural}$; but then the characteristic polynomial is

$$
\begin{aligned}
\operatorname{det}\left(\gamma-x \mathbf{I}_{4}\right) & =\operatorname{det}\left(\zeta \gamma^{\prime}-x \zeta^{2}\right) \\
& \equiv\left(1-x^{2}\right)\left(1+x^{2}\right) \bmod 6
\end{aligned}
$$

From the classification of torsion elements of $\operatorname{Sp}(4, \mathbb{Z})$ and their characteristic polynomials [Ue], it follows that $\gamma$ is conjugate in $\operatorname{Sp}(4, \mathbb{Z})$ to either $\zeta$ or $\zeta[0,1 ; 0]$. Both these are elements of $\Gamma_{6}^{\text {bil }}$ of order 2 ; their fixed loci in $\mathbb{H}_{2}$ are the divisors $\left\{\tau_{2}=0\right\}$ and $\left\{2 \tau_{2}+\left(\tau_{2}^{2}-\tau_{1} \tau_{3}\right)=0\right\}$ respectively (Humbert surfaces of discriminants 1 and 4$)$.

In view of Lemma 1.1, the toroidal (Voronoi, or Igusa) compactification $\left(\mathcal{A}_{6}^{\natural}\right)^{*}$ of $\mathcal{A}_{6}^{\natural}=\Gamma_{6}^{\natural} \backslash \mathbb{H}_{2}$ is smooth, cf. [SC, pp. 276-277]. The action of $\zeta$ on $\mathcal{A}_{6}^{\natural}$ extends to $\left(\mathcal{A}_{6}^{\natural}\right)^{*}$, and the quotient $X_{6}$ is a compactification of $\mathcal{A}_{6}^{\text {bil }}$ whose singularities are isolated ordinary double points or transverse $A_{1}$ singularities. Hence $X_{6}$ has canonical singularities. It agrees with the Voronoi compactification $\left(\mathcal{A}_{6}^{\mathrm{bil}}\right)^{*}$ at least in codimension 1.

\section{$\S 2$. Modular forms and canonical forms}

Gritsenko and Nikulin, in [GN], construct the weight 3 cusp forms

$$
\begin{aligned}
& F_{3}=\operatorname{Lift}\left(\eta^{5}\left(\tau_{1}\right) \vartheta\left(\tau_{1}, 2 \tau_{2}\right)\right) \in \mathfrak{M}_{3}^{*}\left(\Gamma_{6}^{+}, v_{\eta}^{8} \times \operatorname{id}_{H}\right) \\
& F_{3}^{\prime}=\operatorname{Lift}_{-1}\left(\eta^{5}\left(\tau_{1}\right) \vartheta\left(\tau_{1}, 2 \tau_{2}\right)\right) \in \mathfrak{M}_{3}^{*}\left(\Gamma_{6}^{+}, v_{\eta}^{16} \times \operatorname{id}_{H}\right) \\
& F_{3}^{\prime \prime}=\operatorname{Lift}\left(\eta^{3}\left(\tau_{1}\right) \vartheta\left(\tau_{1}, \tau_{2}\right)^{2} \vartheta\left(\tau_{1}, 2 \tau_{2}\right)\right) \in \mathfrak{M}_{3}^{*}\left(\Gamma_{6}^{+}, v_{\eta}^{12} \times \operatorname{id}_{H}\right)
\end{aligned}
$$

for the extended paramodular group $\Gamma_{6}^{+}$, with character $\chi_{D}$ induced from the characters $v_{\eta}^{D} \times \mathrm{id}_{H}$ of the Jacobi group $\mathrm{SL}(2, \mathbb{Z}) \ltimes H(\mathbb{Z})$. Recall (see [GH1], [GN]: for compatibility with [Mu] and other sources we work with the transposes of the groups given in $[\mathrm{GN}]$ ) that $\Gamma_{6}^{+}$is the group generated 
by the paramodular group

$$
\Gamma_{6}=\left\{\gamma \in \operatorname{Sp}(4, \mathbb{Q}) \mid \gamma \in\left(\begin{array}{cccc}
\mathbb{Z} & \mathbb{Z} & \mathbb{Z} & 6 \mathbb{Z} \\
6 \mathbb{Z} & \mathbb{Z} & 6 \mathbb{Z} & 6 \mathbb{Z} \\
\mathbb{Z} & \mathbb{Z} & \mathbb{Z} & 6 \mathbb{Z} \\
\mathbb{Z} & \frac{1}{6} \mathbb{Z} & \mathbb{Z} & \mathbb{Z}
\end{array}\right)\right\}
$$

and the extra involution

$$
V_{6}=\left(\begin{array}{cccc}
0 & 1 / \sqrt{6} & 0 & 0 \\
\sqrt{6} & 0 & 0 & 0 \\
0 & 0 & 0 & \sqrt{6} \\
0 & 0 & 1 / \sqrt{6} & 0
\end{array}\right)
$$

Proposition 2.1. All three of $F_{3}, F_{3}^{\prime}$ and $F_{3}^{\prime \prime}$ are cusp forms, without character, of weight 3 for $\Gamma_{6}^{\text {bil }}$.

Proof. The character is induced from $v_{\eta}^{D} \times \mathrm{id}_{H}$ by the inclusion map $j: \operatorname{SL}(2, \mathbb{Z}) \ltimes H(\mathbb{Z}) \rightarrow \Gamma_{6}^{+}$given by

$$
j:\left(\left(\begin{array}{ll}
a & b \\
c & d
\end{array}\right),[m, n ; k]\right) \longmapsto\left(\begin{array}{cccc}
a & m & c & 0 \\
0 & 1 & 0 & 0 \\
b & n & d & 0 \\
n & k & -m & 1
\end{array}\right) .
$$

For $\gamma \in \mathrm{SL}(2, \mathbb{Z})$ we define $j_{1}(\gamma)=j(\gamma,[0,0 ; 0])$, putting $\gamma$ in the first and third rows and columns in $\operatorname{Sp}(4, \mathbb{Z})$; and similarly $j_{2}(\gamma)$ puts it in the second and fourth.

The character $v_{\eta}^{D} \times \mathrm{id}_{H}$ is trivial on $H(\mathbb{Z})$. In the present cases, where $D=8,16$ or $12, v_{\eta}^{D}$ is trivial on $\pm \Gamma(6)= \pm \operatorname{Ker}(\operatorname{SL}(2, \mathbb{Z}) \rightarrow \operatorname{SL}(2, \mathbb{Z} / 6))$ by $\left[\mathrm{GN}\right.$, Lemma 1.2]. Since $j\left(-\mathbf{I}_{2},[0,0 ; 0]\right)=\zeta$, we see that

$$
\Gamma_{6}^{\mathrm{bil}} \cap j(\mathrm{SL}(2, \mathbb{Z}) \ltimes H(\mathbb{Z})) \subseteq j( \pm \Gamma(6) \ltimes H(\mathbb{Z})) \subseteq \operatorname{Ker} \chi_{D}
$$

for $D=8,12,16$. If $D=8$ or 16 then, since $V_{6}$ and $I=j_{1}\left(\left(\begin{array}{cc}0 & 1 \\ -1 & 0\end{array}\right)\right)$ are in $\Gamma_{6}^{+}$and have even order and the order of $\chi_{D}$ is 3 , we know that $\chi_{D}\left(V_{6}\right)=\chi_{D}(I)=1$. Therefore the element

$$
J_{6}=I V_{6} I V_{6}=\left(\begin{array}{cccc}
0 & 0 & -1 & 0 \\
0 & 0 & 0 & -6 \\
1 & 0 & 0 & 0 \\
0 & 1 / 6 & 0 & 0
\end{array}\right) \in \Gamma_{6}^{+}
$$


is in $\operatorname{Ker} \chi_{D}$. If $D=12$ then $\chi_{12}\left(J_{6}\right)=\chi_{12}\left(I V_{6}\right)^{2}=1$ so again $J_{6} \in \operatorname{Ker} \chi_{D}$. Now we proceed as in [Gr, Lemma 2.2], and show that the group generated by $j(\Gamma(6) \ltimes H(\mathbb{Z}))$ and $J_{6}$ includes $\Gamma_{6}^{\natural}$. To see this, we work with the conjugate groups $\widetilde{\Gamma}_{6}^{\natural}=\nu_{6}\left(\Gamma_{6}^{\natural}\right)$ and $\widetilde{\Gamma}_{6}=\nu_{6}\left(\Gamma_{6}\right)$, where $\nu_{6}$ denotes conjugation by $R_{6}=\operatorname{diag}(1,1,1,6)$. Note that $\nu_{6}\left(J_{6}\right)=R_{6} J_{6} R_{6}^{-1}=\left(\begin{array}{cc}0 & -\mathbf{I}_{2} \\ \mathbf{I}_{2} & 0\end{array}\right)$. If $\tilde{\gamma} \in \widetilde{\Gamma}_{6}^{\natural}$ then its second row $\tilde{\gamma}_{2 *}$ is $(0,1,0,0) \bmod 6$. Suppose first that $\tilde{\gamma}_{22}=1$ and put

$$
\tilde{\beta}=\nu_{6}\left(J_{6}\left[\tilde{\gamma}_{21} / 6, \tilde{\gamma}_{23} / 6 ; \tilde{\gamma}_{24} / 6\right] J_{6}^{-1}\right)=\left(\begin{array}{cccc}
1 & 0 & 0 & \tilde{\gamma}_{23} / 6 \\
\tilde{\gamma}_{21} & 1 & \tilde{\gamma}_{23} & \tilde{\gamma}_{24} \\
0 & 0 & 1 & \tilde{\gamma}_{23} / 6 \\
0 & 0 & 0 & 1
\end{array}\right)
$$

Now $(0,1,0,0) \tilde{\beta}=\tilde{\gamma}_{2 *}$ so the second row of $\tilde{\gamma} \tilde{\beta}^{-1} \in \widetilde{\Gamma}_{6}^{\natural}$ is $(0,1,0,0)$. Such a matrix is in $\nu_{6}(j(\Gamma(6) \ltimes H(\mathbb{Z})))$.

It remains to reduce to the case $\tilde{\gamma}_{22}=1$. Certainly the vector $\tilde{\gamma}_{2 *}$ is primitive, since $\operatorname{det} \tilde{\gamma}=1$, and since $\tilde{\gamma} \in \widetilde{\Gamma}_{6}^{\natural}$ we have $\operatorname{gcd}\left(6, \tilde{\gamma}_{21}, \tilde{\gamma}_{23}\right)=6$. In the proof of [FS, Satz 2.1], it is shown that there are integers $\lambda, \mu$ such that $\tilde{\gamma}^{\prime}=\tilde{\gamma} \nu_{6}\left([\mu, 0 ; 0] J_{6}[0, \lambda ; 0] J_{6}^{-1}\right)$ has $\operatorname{gcd}\left(\tilde{\gamma}_{21}^{\prime}, \tilde{\gamma}_{23}^{\prime}\right)=6$, so the second row of $\tilde{\gamma}^{\prime}$ is $\left(6 x_{1}, 6 x_{2}+1,6 x_{3}, 6 x_{4}\right)$ with $\operatorname{gcd}\left(x_{1}, x_{3}\right)=1$. But then the $(2,2)$-entry of $\tilde{\gamma}^{\prime} \nu_{6}([m, n ; 0])$ is $6\left(m x_{1}+n x_{3}+x_{2}\right)+1$ which is equal to 1 if we choose $m$ and $n$ suitably.

Proposition 2.2. The differential forms $\tilde{\omega}=F_{3} d \tau_{1} \wedge d \tau_{2} \wedge d \tau_{3}, \tilde{\omega}^{\prime}=$ $F_{3}^{\prime} d \tau_{1} \wedge d \tau_{2} \wedge d \tau_{3}$ and $\tilde{\omega}^{\prime \prime}=F_{3}^{\prime \prime} d \tau_{1} \wedge d \tau_{2} \wedge d \tau_{3}$ give rise to canonical forms $\omega, \omega^{\prime}, \omega^{\prime \prime} \in H^{0}\left(K_{X_{6}}\right)$.

Proof. By Proposition 2.1, $\tilde{\omega}, \tilde{\omega}^{\prime}$ and $\tilde{\omega}^{\prime \prime}$ are all $\Gamma_{6}^{\text {bil }}$-invariant, so they give rise to forms $\omega, \omega^{\prime}, \omega^{\prime \prime}$ on $\mathcal{A}_{6}^{\text {bil }}$. Since $F_{3}, F_{3}^{\prime}$ and $F_{3}^{\prime \prime}$ are cusp forms, if any of $\omega, \omega^{\prime}$ and $\omega^{\prime \prime}$ are holomorphic on $\mathcal{A}_{6}^{\text {bil }}$ they extend holomorphically to the cusps of $\left(\mathcal{A}_{6}^{\mathrm{bil}}\right)^{*}$. Since $X_{6}$ agrees with $\left(\mathcal{A}_{6}^{\mathrm{bil}}\right)^{*}$ in codimension 1 and has canonical singularities it follows that these forms can be thought of as 3 -forms on $X_{6}$ holomorphic at infinity. We need to check that $\omega, \omega^{\prime}$ and $\omega^{\prime \prime}$ are holomorphic everywhere. But this is a well-known result of Freitag ([Fr, Satz II.2.6]).

\section{§3. Divisors in the moduli spaces}

In this section we shall describe the canonical divisors $\operatorname{Div}_{X_{6}}(\omega)$, $\operatorname{Div}_{X_{6}}\left(\omega^{\prime}\right)$ and $\operatorname{Div}_{X_{6}}\left(\omega^{\prime \prime}\right)$ in $X_{6}$ and give some detail about the branching locus in $X_{6}$ arising from torsion in $\Gamma_{6}^{\text {bil }}$. 
$\Gamma_{6}^{b i l}$ is a subgroup both of the paramodular group $\Gamma_{6}$ and of $\Gamma_{6}^{+}$. Hence there is a finite morphism $\sigma: \mathcal{A}_{6}^{\mathrm{bil}} \rightarrow \mathcal{A}_{6}^{+}$. We denote the projection map $\mathbb{H}_{2} \rightarrow \mathcal{A}_{6}^{\text {bil }}$ by $\pi_{6}^{\text {bil }}$ and similarly $\pi_{6}, \pi_{6}^{+}$, etc.

For discriminant $\Delta=1,4$ we put

$$
\mathcal{H}_{\Delta}(k)=\left\{\left(\begin{array}{ll}
\tau_{1} & \tau_{2} \\
\tau_{2} & \tau_{3}
\end{array}\right) \in \mathbb{H}_{2} \mid \frac{1}{24}\left(k^{2}-\Delta\right) \tau_{1}+k \tau_{2}+6 \tau_{3}\right\}=0
$$

where $k \in \mathbb{Z}$ is chosen so that $\frac{1}{24}\left(k^{2}-\Delta\right) \in \mathbb{Z}$. The irreducible components of the Humbert surfaces $H_{1}$ and $H_{4}$ of discriminants 1 and 4 in $\mathcal{A}_{6}$ are $\pi_{6}\left(\mathcal{H}_{1}(k)\right)$ and $\pi_{6}\left(\mathcal{H}_{4}(k)\right)$ for $0 \leq k<6$ : the statements of [vdG, Theorem IX.2.4] and of [GH1, Corollary 3.3] are wrong because $\mathcal{H}_{\Delta}(-k)$ is $\Gamma_{t}$-equivalent to $\mathcal{H}_{\Delta}(k)$. Nevertheless the irreducible components of the Humbert surfaces of discriminants 1 and 4 in $\mathcal{A}_{6}^{+}$are as stated in [GN], namely $\pi_{6}^{+}\left(\mathcal{H}_{1}(1)\right)$ and $\pi_{6}^{+}\left(\mathcal{H}_{1}(5)\right)$ for discriminant 1 and $\pi_{6}^{+}\left(\mathcal{H}_{4}(1)\right)$ for discriminant 4 .

The calculation of the divisors uses the product expansion of the modular forms $F_{3}, F_{3}^{\prime}$ and $F_{3}^{\prime \prime}$ given in [GN]. We have chosen to work with the transposes of the matrices given in [GN], so we have to write $q=e^{2 \pi i \tau_{1}}$, $r=e^{2 \pi i \tau_{2} / 6}$ and $s=e^{2 \pi i \tau_{3} / 36}$ for these expansions to be correct. This is because ${ }^{\top} \Gamma_{t}=\operatorname{diag}\left(1, t, 1, t^{-1}\right) \Gamma_{t} \operatorname{diag}\left(1, t^{-1}, 1, t\right)$ (for any $t \in \mathbb{N}$ ), and $\operatorname{diag}\left(1, t, 1, t^{-1}\right):\left(\tau_{1}, \tau_{2}, \tau_{3}\right) \rightarrow\left(\tau_{1}, t \tau_{2}, t^{2} \tau_{3}\right)$. A similar correction is needed in [GH2].

By [GN], equations (4.12)-(4.14), correcting a minor misprint, we have

$$
\begin{aligned}
& F_{3}=\operatorname{Exp}-\operatorname{Lift}\left(5 \phi_{0,3}^{2}-4 \phi_{0,2} \phi_{0,4}\right)=\operatorname{Exp}-\operatorname{Lift}\left(\phi_{3}\right), \\
& F_{3}^{\prime}=\operatorname{Exp}-\operatorname{Lift}\left(\phi_{0,3}^{2}\right)=\operatorname{Exp}-\operatorname{Lift}\left(\phi_{3}^{\prime}\right), \\
& F_{3}^{\prime \prime}=\operatorname{Exp}-\operatorname{Lift}\left(3 \phi_{0,3}^{2}-2 \phi_{0,2} \phi_{0,4}\right)=\operatorname{Exp}-\operatorname{Lift}\left(\phi_{3}^{\prime \prime}\right) .
\end{aligned}
$$

$\left(\phi_{3}, \phi_{3}^{\prime}\right.$ and $\phi_{3}^{\prime \prime}$ are defined by these formulae.)

By [GN, Example 2.3 and Lemma 2.5], we have

$$
\begin{aligned}
& \phi_{0,2}=\left(r^{ \pm 1}+4\right)+q\left(r^{ \pm 3}-8 r^{ \pm 2}-r^{ \pm 1}+16\right)+O\left(q^{2}\right), \\
& \phi_{0,3}=\left(r^{ \pm 1}+2\right)+q\left(-2 r^{ \pm 3}-2 r^{ \pm 2}+2 r^{ \pm 1}+4\right)+O\left(q^{2}\right), \\
& \phi_{0,4}=\left(r^{ \pm 1}+1\right)+q\left(-r^{ \pm 4}-r^{ \pm 3}+r^{ \pm 1}+2\right)+O\left(q^{2}\right),
\end{aligned}
$$

where the notation $r^{ \pm k}$ means $r^{k}+r^{-k}$. 
Proposition 3.1. The divisors in $\mathbb{H}_{2}$ of the cusp forms are

$$
\begin{aligned}
\operatorname{Div}\left(F_{3}\right) & =\left(\pi_{6}^{+}\right)^{-1}\left(\pi_{6}^{+}\left(\mathcal{H}_{1}(1)+5 \mathcal{H}_{1}(5)+\mathcal{H}_{4}(1)\right)\right), \\
\operatorname{Div}\left(F_{3}^{\prime}\right) & =\left(\pi_{6}^{+}\right)^{-1}\left(\pi_{6}^{+}\left(5 \mathcal{H}_{1}(1)+\mathcal{H}_{1}(5)+\mathcal{H}_{4}(1)\right)\right), \\
\operatorname{Div}\left(F_{3}^{\prime \prime}\right) & =\left(\pi_{6}^{+}\right)^{-1}\left(\pi_{6}^{+}\left(3 \mathcal{H}_{1}(1)+3 \mathcal{H}_{1}(5)+\mathcal{H}_{4}(1)\right)\right) .
\end{aligned}
$$

Remark. This corrects the coefficients given in [GN, Example 4.6]: for instance, it is easy to see, by considering the effect of an element of order 2 fixing an Humbert surface, that the coefficients of $\mathcal{H}_{1}(1), \mathcal{H}_{1}(5)$ and $\mathcal{H}_{4}(1)$ must be odd.

Proof. Write $\phi_{3}=\sum f(n, l) q^{n} r^{l}$, and similarly for $\phi_{3}^{\prime}$ and $\phi_{3}^{\prime \prime}$. By [GN, Theorem 2.1], the coefficient of $\pi_{6}^{+}\left(\mathcal{H}_{\Delta}(b)\right)$ in $\mathcal{A}_{6}^{+}$is

$$
m_{\Delta, b}=\sum_{d>0} f\left(d^{2} a, d b\right)
$$

where $b^{2}-24 a=\Delta$. So to calculate $m_{1,1}$ we may take $b=1$ and $a=0$, so $m_{1,1}=\sum_{d>0} f(0, d)$. From the formulae above, $\phi_{3}=\left(r^{ \pm 2}+6\right)+O(q)$, so $m_{1,1}=f(0,2)=1$. Similarly we have $\phi_{3}^{\prime}=\left(r^{ \pm 2}+4 r^{ \pm 1}+6\right)$ so $m_{1,1}^{\prime}=5$ and $\phi_{3}^{\prime \prime}=\left(r^{ \pm 2}+2 r^{ \pm 1}+6\right)$ so $m_{1,1}^{\prime \prime}=3$.

To calculate the coefficients of $\pi_{6}^{+}\left(\mathcal{H}_{4}(1)\right)$ we note that $\mathcal{H}_{4}(1)$ is $\Gamma_{6}^{+}$equivalent to $\mathcal{H}_{4}(2)$, so we may as well work with that and calculate $m_{4,2}$. For this purpose we can take $b=2$ and $a=0$; so $m_{4,2}=\sum_{d>0} f(0,2 d)=1$, and $m_{4,2}^{\prime}=m_{4,2}^{\prime \prime}=1$ also.

To calculate $m_{1,5}$ we take $b=5$ and $a=1$, so $m_{1,5}=\sum_{d>0} f\left(d^{2}, 5 d\right)$. The Fourier coefficient $f(n, l)$ depends only on $24 n-l^{2}$ and on the residue class of $l \bmod 12($ see $[\mathrm{GN}])$; that is, in our case, on $d^{2}$ and on $d \bmod 12$. If $d \not \equiv \pm 1 \bmod 6$ then $5 d \equiv \pm d \bmod 12$, so $f\left(d^{2}, 5 d\right)=f(0, \pm d)$ which is zero unless $d= \pm 2$ or $d=0$. Since we are only interested in $d>0$ the only contribution for $d \not \equiv \pm 1 \bmod 6$ arises from $d=2$, when $f(4,10)=$ $f(0,-2)=1$. If $d \equiv \pm 5 \bmod 12$ then $f\left(d^{2}, 5 d\right)=f\left(\frac{-d^{2}+1}{24}, \pm 1\right)$ which vanishes because $f(n, l)=0$ for $n<0$. If $d \equiv \pm 1 \bmod 12$ then $f\left(d^{2}, 5 d\right)=$ $f\left(\frac{-d^{2}+25}{24}, \pm 5\right)$ which vanishes except possibly when $d=1$. So $m_{1,5}=$ $1+f(1,5)$ and from the expansions of $\phi_{0,2}, \phi_{0,3}$ and $\phi_{0,4}$ we calculate $f(1,5)=4$. Similarly $m_{1,5}^{\prime}=1+f^{\prime}(1,5)=1$ and $m_{1,5}^{\prime \prime}=1+f^{\prime \prime}(1,5)=3$.

Brasch $[\mathrm{Br}]$ has studied the branch locus of $\pi_{t}^{\text {lev }}: \mathbb{H}_{2} \rightarrow \mathcal{A}_{t}^{\text {lev }}$ for all $t$ : for $t \equiv 2 \bmod 4$ the divisorial part has five irreducible components. They 
are $\pi_{6}^{\text {lev }}\left(\mathcal{H}_{\zeta_{i}}\right)$ for $0 \leq i \leq 4$, where $\mathcal{H}_{\zeta_{i}} \subset \mathbb{H}_{2}$ is the fixed locus of $\zeta_{i}$ and

$$
\begin{gathered}
\zeta_{0}=\zeta, \quad \zeta_{1}=\zeta^{\top}[-6,0 ; 0], \quad \zeta_{2}=\left(\begin{array}{cccc}
-7 & 4 & 0 & 0 \\
-12 & 7 & 0 & 0 \\
0 & 0 & -7 & -12 \\
0 & 0 & 4 & 7
\end{array}\right), \\
\zeta_{3}=\zeta[1,0 ; 0], \quad \zeta_{4}=\left(\begin{array}{cccc}
-1 & -1 & 0 & 6 \\
0 & 1 & -6 & 0 \\
0 & 0 & -1 & 0 \\
0 & 0 & -1 & 1
\end{array}\right) .
\end{gathered}
$$

These are all elements of $\Gamma_{6}^{\text {bil }}$. Their fixed loci are

$$
\begin{gathered}
\mathcal{H}_{\zeta_{0}}=\left\{\tau_{2}=0\right\}, \quad \mathcal{H}_{\zeta_{1}}=\left\{6 \tau_{1}-2 \tau_{2}=0\right\}, \quad \mathcal{H}_{\zeta_{2}}=\left\{6 \tau_{1}-7 \tau_{2}+2 \tau_{3}=0\right\}, \\
\mathcal{H}_{\zeta_{3}}=\left\{2 \tau_{2}+\tau_{3}=0\right\}, \quad \mathcal{H}_{\zeta_{4}}=\left\{2 \tau_{2}+\tau_{3}-6=0\right\}
\end{gathered}
$$

of discriminants $1,4,1,4,4$ respectively. Thus three of the components have discriminant 4 and therefore map to $\pi_{6}^{+} \mathcal{H}_{4}(1) \subset \mathcal{A}_{6}^{+}$(they correspond to bielliptic abelian surfaces). $\mathcal{H}_{\zeta_{0}}=\mathcal{H}_{1}(1)$ corresponds to product surfaces $E \times E^{\prime}$ with polarisation given by $\mathcal{O}_{E}(1) \otimes \mathcal{O}_{E^{\prime}}(6)$, and $\mathcal{H}_{\zeta_{2}}$ maps to $\pi_{6}^{+}\left(\mathcal{H}_{1}(5)\right)$, corresponding to abelian surfaces $E \times E^{\prime}$ with polarisation $\mathcal{O}_{E}(2) \otimes \mathcal{O}_{E^{\prime}}(3)$.

Proposition 3.2. The branch locus of $\pi_{6}^{\mathrm{bil}}: \mathbb{H}_{2} \rightarrow \mathcal{A}_{6}^{\mathrm{bil}}$ has seven irreducible components, each with branching of order 2 . They are $\pi_{6}^{\mathrm{bil}}\left(\mathcal{H}_{\zeta_{i}}\right)$ and two other components $\pi_{6}^{\text {bil }}\left(\mathcal{H}_{\zeta_{1}^{\prime}}\right), \pi_{6}^{\text {bil }}\left(\mathcal{H}_{\zeta_{1}^{\prime \prime}}\right)$, which are equivalent to $\pi_{6}^{\text {bil }}\left(\mathcal{H}_{\zeta_{1}}\right)$ in $\mathcal{A}_{6}^{\text {lev }}$.

Proof. It follows from Lemma 1.1 that the branch locus consists of divisors only and that the branching is of order 2 .

Write $G=\Gamma_{6}^{\text {lev }} \triangleright H=\Gamma_{6}^{\text {bil }}$ and let $G$ act on $\Omega=G / H \cong \operatorname{PSL}(2, \mathbb{Z} / 6)$. By [Br, Corollary 1.3], the number of irreducible divisors in $\mathcal{A}_{6}^{\text {bil }}$ mapping to $\pi_{6}^{\text {lev }}\left(\mathcal{H}_{\zeta_{i}}\right)$, which is equal to the number of $H$-conjugacy classes in the $G$-conjugacy class of $\zeta_{i}$, is $\left|G: H . C_{G}\left(\zeta_{i}\right)\right|$. (If $\xi \in G$ for some group $G$ then $C_{G}(\xi)$ denotes the centraliser of $\xi$ in $G$.) Moreover, for fixed $i$, these divisors are permuted transitively by $\Omega$ so they all have the same branching behaviour: $\pi_{6}^{\text {bil }}$ is branched of order 2 above each one.

$\left|G: H . C_{G}\left(\zeta_{i}\right)\right|=\left|G / H: C_{G}\left(\zeta_{i}\right) /\left(H \cap C_{G}\left(\zeta_{i}\right)\right)\right|$, which is the index of the image of $C_{G}\left(\zeta_{i}\right)$ in $\Omega$. For $i=0,1,2,3$ the centraliser $C_{\operatorname{Sp}(4, \mathbb{Q})}\left(\zeta_{i}\right)$ is described in [Br, Lemma 2.1], and $C_{G}\left(\zeta_{i}\right)=C_{\mathrm{Sp}(4, \mathbb{Q})}\left(\zeta_{i}\right) \cap G$. 
For $\zeta_{0}$, if $\gamma \in \operatorname{PSL}(2, \mathbb{Z} / 6) \cong \Omega$ and $\tilde{\gamma} \in \operatorname{SL}(2, \mathbb{Z})$ is some lift of $\gamma$ then $j(\tilde{\gamma},[0,0 ; 0]) \in C_{G}\left(\zeta_{0}\right)$ so the index is 1 .

For $\zeta_{1}$, if $\gamma=\left(\begin{array}{ll}a & b \\ c & d\end{array}\right) \in \operatorname{PSL}(2, \mathbb{Z} / 6)$ and $b$ is even then

$$
\left(\begin{array}{cccc}
\tilde{a} & 0 & \tilde{b} & 3 \tilde{b} \\
3(\tilde{a}-1) & 1 & 3 \tilde{b} & 0 \\
\tilde{c} & 0 & \tilde{d} & 3(\tilde{d}-1) \\
0 & 0 & 0 & 1
\end{array}\right) \in C_{G}\left(\zeta_{1}\right)
$$

for a lift $\tilde{\gamma}$; and this is a necessary condition for such an element to exist since if $\beta=\beta_{i j} \in C_{G}\left(\zeta_{1}\right)$ then $3 \beta_{13} \equiv 0 \bmod 6$. So $C_{G}\left(\zeta_{1}\right) /\left(C_{G}\left(\zeta_{1}\right) \cap\right.$ $H) \subset \operatorname{PSL}(2, \mathbb{Z} / 6)$ is the reduction $\bmod 6$ of ${ }^{\top} \Gamma_{0}(2)$, i.e. the preimage of $\left\{\left(\begin{array}{ll}a & 0 \\ c & d\end{array}\right) \in \mathrm{SL}(2, \mathbb{Z} / 2)\right\}$, which is of index 3 because it is the stabiliser of $(1,0)$ when $\mathrm{SL}(2, \mathbb{Z} / 2)$ acts as the symmetric group on the nonzero vectors in $\mathbb{F}_{2}^{2}$.

For $\zeta_{2}$, any two elements $\gamma, \gamma^{*} \in \mathrm{SL}(2, \mathbb{Q})$ determine an element $\beta\left(\gamma, \gamma^{*}\right)$ $\in C_{\mathrm{Sp}(4, \mathbb{Q})}$ (see [Br, Lemma 2.1] and the preceding discussion), namely

$$
\beta\left(\gamma, \gamma^{*}\right)=\left(\begin{array}{cccc}
4 \gamma_{11}-3 \gamma_{11}^{*} & -2 \gamma_{11}+2 \gamma_{11}^{*} & 4 \gamma_{12}+\gamma_{12}^{*} & 6 \gamma_{12}+2 \gamma_{12}^{*} \\
6 \gamma_{11}-6 \gamma_{11}^{*} & -3 \gamma_{11}+4 \gamma_{11}^{*} & 6 \gamma_{12}+2 \gamma_{12}^{*} & 9 \gamma_{12}+4 \gamma_{12}^{*} \\
4 \gamma_{21}+9 \gamma_{21}^{*} & -2 \gamma_{21}-6 \gamma_{21}^{*} & 4 \gamma_{22}-3 \gamma_{22}^{*} & 6 \gamma_{22}-6 \gamma_{22}^{*} \\
-2 \gamma_{21}-6 \gamma_{21}^{*} & \gamma_{21}+4 \gamma_{21}^{*} & -2 \gamma_{22}+2 \gamma_{22}^{*} & -3 \gamma_{22}+4 \gamma_{22}^{*}
\end{array}\right) .
$$

In particular we choose

$$
\beta=\beta\left(\left(\begin{array}{ll}
3 & 4 \\
2 & 3
\end{array}\right),\left(\begin{array}{cc}
10 & 9 \\
11 & 10
\end{array}\right)\right)=\left(\begin{array}{cccc}
-18 & 14 & 25 & 42 \\
-42 & 31 & 42 & 72 \\
107 & -70 & -18 & -42 \\
-70 & 46 & -14 & 31
\end{array}\right)
$$

and

$$
\beta^{\prime}=\beta\left(\left(\begin{array}{cc}
11 & 4 \\
8 & 3
\end{array}\right),\left(\begin{array}{ll}
7 & 9 \\
3 & 4
\end{array}\right)\right)=\left(\begin{array}{cccc}
23 & -8 & 25 & 42 \\
24 & -5 & 42 & 72 \\
59 & -34 & 0 & -6 \\
-34 & 20 & -6 & 7
\end{array}\right)
$$

$\beta$ and $\beta^{\prime}$ both belong to $\Gamma_{6}^{\text {lev }}$, and their images in $\operatorname{PSL}(2, \mathbb{Z} / 6)$ are $\left(\begin{array}{cc}0 & 1 \\ -1 & 0\end{array}\right)$ and $\left(\begin{array}{ll}-1 & 1 \\ -1 & 0\end{array}\right)$. These two elements generate $\operatorname{PSL}(2, \mathbb{Z} / 6)$ because their lifts generate $\operatorname{SL}(2, \mathbb{Z})$, so the index we want is 1 . 
For $\zeta_{3}$, as for $\zeta_{0}, j(\tilde{\gamma},[0,0 ; 0]) \in C_{G}\left(\zeta_{3}\right)$ so the index is 1 .

For $\zeta_{4}$, note that $\zeta_{4}={ }^{\top}[0,0 ; 6] \zeta_{3}\left({ }^{\top}[0,0 ; 6]\right)^{-1}$ so $C_{\mathrm{Sp}(4, \mathbb{Q})}\left(\zeta_{4}\right)={ }^{\top}[0,0 ; 6]$ $C_{\mathrm{Sp}(4, \mathbb{Q})}\left(\zeta_{3}\right)\left(^{\top}[0,0 ; 6]\right)^{-1}$. It happens that ${ }^{\top}[0,0 ; 6] j(\tilde{\gamma},[0,0 ; 0])\left({ }^{\top}[0,0 ; 6]\right)^{-1}$ $=j(\tilde{\gamma},[0,0 ; 0])$, so again the index is 1 .

Next we look at the boundary divisors of $X_{6}$. These correspond to 1-dimensional subspaces of $\mathbb{Q}^{4}$ up to the action of $\Gamma_{6}^{\text {bil }}$. We may think of such a space as being given by a unique, up to sign, primitive vector $\mathbf{v}=\left(v_{1}, v_{2}, v_{3}, v_{4}\right) \in \mathbb{Z}^{4}$. It is shown in [FS, Satz 2.1], that the $\Gamma_{6}$-orbit of $\mathbf{v}$ is determined by $r=\operatorname{gcd}\left(6, v_{1}, v_{3}\right)$, so $\mathcal{A}_{6}$ has four corank 1 cusps (or boundary divisors in the toroidal compactification). However, the cusps $r=1$ and $r=6$ are interchanged by $V_{6}$, as are the cusps $r=2$ and $r=3$, so $\mathcal{A}_{6}^{+}$has just two corank 1 cusps. Since $F_{3}, F_{3}^{\prime}$ and $F_{3}^{\prime \prime}$ are modular forms (with character) for $\Gamma_{6}^{+}$, the order of vanishing of any of them at a cusp of $X_{6}$ given by $\mathbf{v}$ depends only on which cusp of $\mathcal{A}_{6}^{+}$it lies over, i.e. on whether $r$ is or is not a proper divisor of 6 .

We write $D_{1}$ for the divisor in $X_{6}$ which is the sum of all the boundary components with $r=1$ or $r=6$, and $D_{2}$ for the sum of all the components with $r=2$ or $r=3$. By modifying the argument of [FS, Satz 2.1] as in [Sa], it can be shown that $D_{1}$ has 28 irreducible components and $D_{2}$ has 12 , but we shall not make any use of this.

TheOREM 3.3. The divisors of $\omega, \omega^{\prime}$ and $\omega^{\prime \prime}$ in $X_{6}$ are

$$
\begin{aligned}
\operatorname{Div}_{X_{6}}(\omega) & =4 \pi_{6}^{\mathrm{bil}}\left(\mathcal{H}_{\zeta_{2}}\right)+D_{1}+D_{2}, \\
\operatorname{Div}_{X_{6}}\left(\omega^{\prime}\right) & =4 \pi_{6}^{\mathrm{bil}}\left(\mathcal{H}_{\zeta_{0}}\right)+3\left(D_{1}+D_{2}\right), \\
\operatorname{Div}_{X_{6}}\left(\omega^{\prime \prime}\right) & =2 \pi_{6}^{\mathrm{bil}}\left(\mathcal{H}_{\zeta_{0}}\right)+2 \pi_{6}^{\mathrm{bil}}\left(\mathcal{H}_{\zeta_{2}}\right)+2\left(D_{1}+D_{2}\right) .
\end{aligned}
$$

Proof. If $\pi_{6}^{\text {bil }}$ is branched along the irreducible divisors $B_{\alpha}$ with ramification index $e_{\alpha}$, then $d \tau_{1} \wedge d \tau_{3} \wedge d \tau_{3}$ acquires poles of order $e_{\alpha} / 2$ along $B_{\alpha}$. So by Proposition 3.1

$$
\begin{aligned}
\operatorname{Div}_{X_{6}}(\omega) & =\sigma^{-1} \pi_{6}^{+}\left(\mathcal{H}_{1}(1)+5 \mathcal{H}_{1}(5)+\mathcal{H}_{4}(1)\right)-\frac{1}{2} \sum e_{\alpha} B_{\alpha}+D \\
\operatorname{Div}_{X_{6}}\left(\omega^{\prime}\right) & =\sigma^{-1} \pi_{6}^{+}\left(5 \mathcal{H}_{1}(1)+\mathcal{H}_{1}(5)+\mathcal{H}_{4}(1)\right)-\frac{1}{2} \sum e_{\alpha} B_{\alpha}+D^{\prime} \\
\operatorname{Div}_{X_{6}}\left(\omega^{\prime \prime}\right) & =\sigma^{-1} \pi_{6}^{+}\left(3 \mathcal{H}_{1}(1)+3 \mathcal{H}_{1}(5)+\mathcal{H}_{4}(1)\right)-\frac{1}{2} \sum e_{\alpha} B_{\alpha}+D^{\prime \prime}
\end{aligned}
$$


where $D, D^{\prime}$ and $D^{\prime \prime}$ are effective divisors supported on the boundary $X_{6} \backslash$ $\mathcal{A}_{6}^{\text {bil }}$. The form of the branch locus part of the divisors follows now from Proposition 3.2 and the discriminants of $\mathcal{H}_{\zeta_{i}}$.

It remains to calculate the vanishing orders of the forms at each boundary divisor. For each form, we need only consider two boundary components, one from $D_{1}$ and one from $D_{2}$. We use the components $D\left(\mathbf{v}_{1}\right), D\left(\mathbf{v}_{2}\right)$ corresponding to $\mathbf{v}_{1}=(0,0,1,0)$ and $\mathbf{v}_{2}=(0,0,2,1)$. The first step in constructing the toroidal compactification near $D\left(\mathbf{v}_{1}\right)$ is to take a quotient by the lattice $P_{\mathbf{v}_{1}}^{\prime}\left(\Gamma_{6}^{\text {bil }}\right.$ ) (see for instance [GH2, pp. 925-926] or for a full explanation [HKW, Section I.3D]). As in [HKW, Proposition I.3.98], $P_{\mathbf{v}_{1}}^{\prime}\left(\Gamma_{6}^{\text {bil }}\right)$ is generated by $j_{1}\left(\left(\begin{array}{ll}1 & 6 \\ 0 & 1\end{array}\right)\right)$; so a local equation for $D\left(\mathbf{v}_{1}\right)$ at a general point is $t_{1}=0$, where $t_{1}=e^{2 \pi i \tau_{1} / 6}=q^{1 / 6}$. Using the values of $f(0, l)$ calculated above and the Fourier expansion given in [GN, Theorem 2.1], we see that the expansions of $F_{3}, F_{3}^{\prime}$ and $F_{3}^{\prime \prime}$ begin $q^{1 / 3} r s^{2}, q^{2 / 3} r^{3} s^{4}$ and $q^{1 / 2} r^{2} s^{3}$ respectively, so their orders of vanishing along $D_{1}$ are 2, 4 and 3 . The form $d \tau_{1} \wedge d \tau_{2} \wedge d \tau_{3}$ contributes a simple pole at the boundary so the coefficients of $D_{1}$ in the divisors of $\omega, \omega^{\prime}$ and $\omega^{\prime \prime}$ are 1,3 and 2 .

We put

$$
\theta=\left(\begin{array}{cccc}
1 & -1 & 0 & 0 \\
-1 & 2 & 0 & 0 \\
0 & 0 & 2 & 1 \\
0 & 0 & 1 & 1
\end{array}\right) \in \operatorname{Sp}(4, \mathbb{Z})
$$

so that $\mathbf{v}_{2}=\mathbf{v}_{1} \theta$. Then $\mathcal{P}_{\mathbf{v}_{2}}=\theta^{-1} \mathcal{P}_{\mathbf{v}_{1}} \theta$ (where, as in [HKW], $\mathcal{P}_{\mathbf{v}}$ denotes the stabiliser of $\mathbf{v}$ in $\operatorname{Sp}(4, \mathbb{Q}))$, and from this one readily calculates that

$$
P_{\mathbf{v}_{2}}^{\prime}\left(\Gamma_{6}^{\mathrm{bil}}\right)=\left\{\left(\begin{array}{cccc}
1 & 0 & 4 n & 2 n \\
0 & 1 & 2 n & n \\
0 & 0 & 1 & 0 \\
0 & 0 & 0 & 1
\end{array}\right) \mid n \equiv 0 \bmod 36\right\}
$$

So the cusp $D_{2}$ is given by $t_{2}=0$, where $t_{2}=e^{2 \pi i\left(\tau_{1} / 144+\tau_{2} / 72+\tau_{3} / 36\right)}=$ $q^{1 / 144} r^{1 / 12} s$. The number of times this term divides the expressions for $F_{3}$, $F_{3}^{\prime}$ and $F_{3}^{\prime \prime}$ is in fact equal to the power of $s$ that occurs, namely 2,4 and 3 respectively; so we get the same orders of vanishing along $D_{2}$ as along $D_{1}$.

This calculation shows directly (without appealing to Freitag's result in $[\mathrm{Fr}])$ that $\omega, \omega^{\prime}$ and $\omega^{\prime \prime}$ are all holomorphic. 
Remark. Notice that $\operatorname{Div}_{X_{6}}(\omega)+\operatorname{Div}_{X_{6}}\left(\omega^{\prime}\right)=2 \operatorname{Div}_{X_{6}}\left(\omega^{\prime \prime}\right)$, reflecting the fact (easily seen from $[\mathrm{GN}]$ ) that $F_{3} F_{3}^{\prime}=\left(F_{3}^{\prime \prime}\right)^{2}$.

Theorem A now follows at once from the following observation.

Proposition 3.4. $\omega, \omega^{\prime}$ and $\omega^{\prime \prime}$ are linearly independent elements of $H^{0}\left(K_{X_{6}}\right)$.

Proof. Suppose that $\lambda \omega+\lambda^{\prime} \omega^{\prime}+\lambda^{\prime \prime} \omega^{\prime \prime}=0$. At a general point of $\pi_{6}^{\text {bil }}\left(\mathcal{H}_{\zeta_{0}}\right), \omega^{\prime}$ and $\omega^{\prime \prime}$ vanish but $\omega$ does not. Therefore $\lambda=0$. Similarly $\lambda^{\prime}=0$, considering a general point of $\pi_{6}^{\text {bil }}\left(\mathcal{H}_{\zeta_{2}}\right)$. Finally, $\lambda^{\prime \prime} \neq 0$ because $F_{3}^{\prime \prime}$ is not identically zero.

We want to remark that $\kappa\left(\mathcal{A}_{6}^{\text {bil }}\right) \geq 1$ can be deduced from the existence of $\omega^{\prime}$ alone. The divisor $\operatorname{Div}_{X_{6}}\left(\omega^{\prime}\right)$ is effective and $\pi_{6}^{\text {bil }}\left(\mathcal{H}_{\zeta}\right) \subset$ Supp $\operatorname{Div}_{X_{6}}\left(\omega^{\prime}\right)$. Since $X_{6}$ has canonical singularities, $K$ is effective on any smooth model of $X_{6}$, and hence also on any minimal model $X_{6}^{\prime}$ of $X_{6}$. Any surfaces contracted by the birational map $X_{6} \rightarrow X_{6}^{\prime}$ must be birationally ruled. But $\pi_{6}^{\text {bil }}\left(\mathcal{H}_{\zeta}\right)$ is not birationally ruled: it is isomorphic to $X(6) \times X(6)$, since $\mathcal{H}_{\zeta}$ is isomorphic to $\mathbb{H} \times \mathbb{H}$ and is preserved by the subgroup $\Gamma(6) \times \Gamma(6)$ embedded in $\Gamma_{6}^{\text {bil }}$ by $\left(j_{1}, j_{2}\right)$. Thus its closure is birationally an abelian surface, since $X(6)$ has genus 1 . So the canonical divisor of $X_{6}^{\prime}$ is effective and nontrivial; so, by abundance, some multiple of it moves and therefore $\kappa\left(\mathcal{A}_{6}^{\mathrm{bil}}\right) \geq 1$.

\section{REFERENCES}

[SC] A. Ash, D. Mumford, M. Rapoport and Y. Tai, Smooth Compactification of Locally Symmetric Varieties, Math. Sci. Press, Brookline, Mass, 1975.

[Br] H.-J. Brasch, Branch points in moduli spaces of certain abelian surfaces, Abelian Varieties, Egloffstein 1993, de Gruyter, Berlin (1995), pp. 27-54.

[Bo] L. A. Borisov, A finiteness theorem for subgroups of $\operatorname{Sp}(4, \mathbb{Z})$, Algebraic geometry, 9, J. Math. Sci. (New York), 94 (1999), 1073-1099.

[FS] M. Friedland and G. K. Sankaran, Das Titsgebäude von Siegelschen Modulgruppen vom Geschlecht 2, Math. Abh. Sem. Univ. Hamburg, 71 (2001), 49-68.

[Fr] E. Freitag, Siegelsche Modulfunktionen, Grundlehren der mathematischen Wissenschaften Bd. 254, Springer-Verlag, Berlin, 1983.

[vdG] G. van der Geer, Hilbert Modular Surfaces, Ergebnisse der Mathematik und ihrer Grenzgebiete Bd. 16, Springer-Verlag, Berlin-New York, 1988.

[Gr] V. Gritsenko, Irrationality of the moduli spaces of polarized abelian surfaces, Abelian Varieties, Egloffstein 1993, de Gruyter, Berlin (1995), pp. 63-84. 
[GH1] V. Gritsenko and K. Hulek, Minimal Siegel modular threefolds, Math. Proc. Cambridge Philos. Soc., 123 (1998), 461-485.

[GH2] V. Gritsenko and K. Hulek, The modular form of the Barth-Nieto quintic, Internat. Math. Res. Notices, 17 (1999), 915-937.

[GN] V. Gritsenko and V. Nikulin, Automorphic forms and Lorentzian Kac-Moody Algebras II, Internat. J. Math., 9 (1998), 201-275.

[HKW] K. Hulek, C. Kahn and S. Weintraub, Moduli spaces of abelian surfaces: Compactification, degenerations and theta functions, de Gruyter, 1993.

$[\mathrm{Mu}] \quad$ S. Mukai, Moduli of abelian surfaces, and regular polyhedral groups, Moduli of algebraic varieties and the monster, Sapporo 1999 (I. Nakamura, ed.), pp. 68-74.

[Sa] G. K. Sankaran, Abelian surfaces with odd bilevel structure, preprint 2002, math.AG/0209192.

[Ue] K. Ueno, On fibre spaces of normally polarized abelian varieties of dimension 2. II. Singular fibres of the first kind, J. Fac. Sci. Univ. Tokyo Sect. IA Math., 19 (1972), 163-199.

G. K. Sankaran

Department of Mathematical Sciences

University of Bath

Bath BA2 7AY

England

gks@maths . bath .ac.uk

J. G. Spandaw

Institut für Mathematik

Universität Hannover

Postfach 6009

D-30060 Hannover

Germany

spandaw@math . uni-hannover.de 